\title{
Robust Quantum Control in Games: an Adversarial Learning Approach
}

\author{
Xiaozhen Ge and Haijin Ding \\ Department of Automation, Tsinghua University, Beijing 100084, China
}

Herschel Rabit2*
Department of Chemistry, Princeton University, Princeton, New Jersey 08544, USA

Rebing $\mathrm{Wv}{ }^{\dagger}$

Department of Automation, Tsinghua University, Beijing 100084, China and

Beijing National Research Center for Information Science and Technology, Beijing 100084, China

\begin{abstract}
High-precision operation of quantum computing systems must be robust to uncertainties and noises in the quantum hardware. In this paper, we show that through a game played between the uncertainties (or noises) and the controls, adversarial uncertainty samples can be generated to find highly robust controls through the search for Nash equilibria (NE). We propose a broad family of adversarial learning algorithms, namely a-GRAPE algorithms, which include two effective learning schemes referred to as the best-response approach and the better-response approach within the game-theoretic terminology, providing options for rapidly learning robust controls. Numerical experiments demonstrate that the balance between fidelity and robustness depends on the details of the chosen adversarial learning algorithm, which can effectively lead to a significant enhancement of control robustness while attaining high fidelity.
\end{abstract}

PACS numbers:

\section{INTRODUCTION}

Recent experimental breakthroughs in quantum computing have signaled its commercialization in the foreseeable future 1 3. The demands involved require highly precise and stable control techniques for deterministic implementation of quantum gates [4. Generally, high-precision is relatively easy to achieve if a well characterized model is available, for example, using the highly efficient GRAPE (gradient ascent pulse engineering) algorithm [5] and methodologies based on reinforcement learning 6. The real challenge is to maintain high precision in the presence of realistic uncertainties and noises in the model, i.e., finding both high-precision and robust controls. In the literature, a variety of proposals have been put forth for either online or offline searches for robust quantum controls, e.g., stimulated Raman adiabatic passage working to overcome pulse shape errors $7 \sqrt{9}$, dynamical decoupling to fight against decoherence noises [10 13, and differential evolution [14, 15] as well as ensemble-based algorithms [5, 16] aiming to

*Electronic address: hrabitz@princeton.edu

$\dagger$ Electronic address: rbwu@tsinghua.edu.cn deal with a variety of noises and uncertainties .

Recently, a class of robust control design methods have been proposed 17-21. It was shown that, by Monte Carlo sampling of the uncertainties or noises, the GRAPE algorithm can be exploited to effectively improve the robustness against linewidth broadening [17/19], amplitude errors of control fields [18, 19], coupling uncertainty 21, and clock noises 22].

Most of the above approaches are based on improving robustness of a quantum control protocol quantified by the average performance with respect to the system uncertainties or noises to be suppressed. This measure is relatively easy to evaluate and hence to optimize. However, it is not the unique choice, nor necessarily the best. For example, fighting against the worst-case performance was widely adopted in the control of classical systems 23 26, which leads to a class of min-max problems. The optimization with respect to such an objective can effectively reduce the risk of failure, which is demanded by fault-tolerant quantum computation. The worst-case optimization for robust quantum controls was first formulated in the control of molecular systems against disturbance 27, 28, and a theoretical analysis was later done on the robust performance via the Dyson expansion 29]. However, there are very few algo- 
rithms for efficiently solving the associated minmax problem. In 3032 , the so-called $H_{\infty}$ approach was applied to a class of linear quantum systems in the Heisenberg picture. In 33, sequential convex programming (SCP) was proposed for solving the worst-case robust optimization problem in a single-qubit system, which decomposes the min-max problem into a sequence of convex optimization procedures.

Since the min-max problem can be taken as an adversarial game between the control and the uncertainties (or noises) that attempt to affect the objective of the quantum system (e.g., state or gate infidelity) in opposite directions, one can introduce game-theoretic learning algorithms that seek Nash equilibria (NE) [34. Even if the Nash equilibrium does not exist or is hard to be reached, the robustness of the control can still be enhanced during the learning process. As the adversarial game has a close relationship with both the fidelity and robustness, the game-based learning process can be adjusted flexibly to make a balance between the two performance indexes, forming a broad family of adversarial learning algorithms. A successful paradigm is the GAN (Generative Adversarial Nets) model in deep learning 35-38 for generative learning tasks, where a large number of applications show that the learning model can be trained to be more generalizable by actively generating adversarial samples produced by a discriminator neural network.

In this paper, we will extend game-theoretic ideas to the design of robust quantum controls, starting with the worst-case system disturbance. The remainder of this paper will be arranged as follows. Sec. II presents a game-theoretic analysis of the robust control problem. In Sec. III, we introduce several adversarial learning control algorithms based on the dynamic processes in the game theory of learning and evolution. In Sec. IV the effectiveness of the proposed algorithms is illustrated through simulations of the control design in two representive examples. Finally, concluding remarks are made in Sec. V.

\section{THE ZERO-SUM GAME BETWEEN CONTROL AND UNCERTAINTY}

Consider an $N$-dimensional quantum control system whose unitary propagator obeys the fol- lowing Schrödinger equation:

$$
\dot{U}(t ; \boldsymbol{u}, \boldsymbol{\epsilon})=-i H[t ; \boldsymbol{u}, \boldsymbol{\epsilon}] U(t ; \boldsymbol{u}, \boldsymbol{\epsilon})
$$

where $U(\cdot) \in \mathbb{C}^{N \times N}$ represents the quantum gate operation with the initial value being the identity matrix. The system's Hamiltonian $H[t ; \boldsymbol{u}, \boldsymbol{\epsilon}]$ is dependent on a vector of control parameters $\boldsymbol{u}$ (e.g., in-phase and quadrature amplitudes, or phases and amplitudes of laser pulses in the frequencydomain) and a vector of uncertainty parameters $\boldsymbol{\epsilon}$ (e.g., environmental noises or imprecisely identified parameters).

Let $U_{f}$ be the target gate operation and

$$
L[\boldsymbol{u}, \boldsymbol{\epsilon}]=N^{-2}\left\|U(T ; \boldsymbol{u}, \boldsymbol{\epsilon})-U_{f}\right\|^{2}
$$

be the infidelity of the controlled gate under the control $\boldsymbol{u}$ and the uncertainty $\boldsymbol{\epsilon}$, where $\|\cdot\|$ is the Frobenius norm. The robustness objective is to find a control $\boldsymbol{u}$ under which $L[\boldsymbol{u}, \boldsymbol{\epsilon}]$ is as small as possible for as many as possible values of the uncertainty $\boldsymbol{\epsilon}$.

A straightforward approach adopted in most existing studies is to minimize the average infidelity, i.e.,

$$
\mathbf{E}_{\boldsymbol{\epsilon}} L[\boldsymbol{u}, \boldsymbol{\epsilon}]=\int_{\boldsymbol{\epsilon}} L[\boldsymbol{u}, \boldsymbol{\epsilon}] p_{0}(\boldsymbol{\epsilon}) \mathrm{d} \boldsymbol{\epsilon}
$$

where $p_{0}(\boldsymbol{\epsilon})$ is the probability density distribution of $\boldsymbol{\epsilon}$. This objective is relatively easy to estimate and thus to optimize, but the resulting control may not be able to dictate all possible cases of uncertainties due to a lack of control over the variance of the infidelity (i.e., the infidelity can be high over certain small ranges of uncertainties even if the average infidelity is low).

To reduce the risk of encountering high infidelity, we consider the worst-case performance instead of the average performance, which leads to the following min-max problem:

$$
\min _{\boldsymbol{u}} \max _{\boldsymbol{\epsilon}} L[\boldsymbol{u}, \boldsymbol{\epsilon}]
$$

that does not rely on the probability density distribution of $\epsilon$. Once the worst-case infidelity is below the desired threshold value, the risk of high infidelity can be effectively reduced.

From a game-theoretic point of view, the optimization process can be taken as a zero-sum game between two players, the control $\boldsymbol{u}$ and the uncertainty $\boldsymbol{\epsilon}$, in which $\boldsymbol{u}$ attempts to reduce the gate 
infidelity while $\boldsymbol{\epsilon}$ tries to increase it. A robust control is thus naturally associated with the NE point $\left(\boldsymbol{u}^{*}, \boldsymbol{\epsilon}^{*}\right)$ of the game 34, at which each player is unable to go any further by tuning merely $\boldsymbol{u}$ or merely $\epsilon$. That is to say, it holds that

$$
\boldsymbol{u}^{*}=\arg \min _{\boldsymbol{u}} L\left[\boldsymbol{u}, \boldsymbol{\epsilon}^{*}\right] \text { and } \boldsymbol{\epsilon}^{*}=\arg \max _{\boldsymbol{\epsilon}} L\left[\boldsymbol{u}^{*}, \boldsymbol{\epsilon}\right] .
$$

A standard approach to search for such a strategy profile is to alternately minimize (with respect to $\boldsymbol{u}$ ) and maximize (with respect to $\boldsymbol{\epsilon}$ ) the infidelity. Even if the NE does not exist or is hard to be reached, the robustness of the control may still be enhanced during the process of fighting against the so-called adversarial samples of $\epsilon$ that yield the worst performance. In this spirit, a family of learning algorithms can be devised in which the uncertainty parameters play a more active role instead of just being averaged out. Since in this work the gradient-based GRAPE algorithm is always applied in the minimization process, the adversarial learning algorithms to be presented below will be termed as a-GRAPE, where "a" stands for "adversarial".

\section{THE DESIGN OF ADVERSARIAL LEARNING CONTROL ALGORITHMS}

In this section, we will propose two types of aGRAPE algorithms, namely the best-response and better-response approaches, for training highly robust controls via active selection of adversarial samples.

\section{A. Best-response approach}

The simplest NE-seeking approach consists of rounds of alternate optimization with the control and the uncertainty. Suppose that we have obtained an adversarial sample $\boldsymbol{\epsilon}^{(k)}$ subject to the optimal control $\boldsymbol{u}^{(k)}$ in the $k$-th round. In the $(k+1)$-th round, we first minimize $L\left[\boldsymbol{u}, \boldsymbol{\epsilon}^{(k)}\right]$ with respect to $\boldsymbol{u}$ using the GRAPE algorithm, which updates the control by $\boldsymbol{u}^{(k+1)}$. Then, we update the adversarial sample of uncertainty parameters by $\boldsymbol{\epsilon}^{(k+1)}$ that maximizes $L\left[\boldsymbol{u}^{(k+1)}, \boldsymbol{\epsilon}\right]$ with respect to $\epsilon$.

Utilizing game theory terminology, we call such an adversarial learning process a best-response approach because each player chooses its best strategy against the opponent 39 . However, for most robust quantum control problems, the bestresponse strategy can hardly reach a pure NE. This conclusion follows from the facts that a NE requires $L\left[\boldsymbol{u}^{*}, \boldsymbol{\epsilon}\right]=0$ for all admissible $\boldsymbol{\epsilon}$, as the control is assumed to have fully adequate resources (e.g., bandwidth) such that $\min _{\boldsymbol{u}} L[\boldsymbol{u}, \boldsymbol{\epsilon}]=0$ for any $\boldsymbol{\epsilon}$, and that this condition is hard to satisfy in practice unless $\epsilon$ is only allowed to vary over a very small domain. Since a min-max problem possesses a NE when the minimization problem is convex and maximization problem is concave, a viable strategy is to seek a mixed NE in a enlarged domain, where the uncertainty $\epsilon$ is allowed to adopt mixed strategies, i.e., instead of picking a single adversarial sample, we generate a distribution of adversarial samples. Mathematically, this leads to the following min-max problem:

$$
\min _{\boldsymbol{u}} \max _{p(\boldsymbol{\epsilon}) \in \mathcal{P}} \int L[\boldsymbol{u}, \boldsymbol{\epsilon}] p(\boldsymbol{\epsilon}) \mathrm{d} \boldsymbol{\epsilon},
$$

where the maximization is performed over the space of probability density distributions $\mathcal{P}$. The mixed NE $\left(\boldsymbol{u}^{*}, p^{*}(\boldsymbol{\epsilon})\right)$ satisfies

$$
\begin{aligned}
\boldsymbol{u}^{*} & =\arg \min _{\boldsymbol{u}} \int L[\boldsymbol{u}, \boldsymbol{\epsilon}] p^{*}(\boldsymbol{\epsilon}) \mathrm{d} \boldsymbol{\epsilon}, \\
p^{*}(\boldsymbol{\epsilon}) & =\arg \max _{p(\boldsymbol{\epsilon})} \int L\left[\boldsymbol{u}^{*}, \boldsymbol{\epsilon}\right] p(\boldsymbol{\epsilon}) \mathrm{d} \boldsymbol{\epsilon} .
\end{aligned}
$$

The advantage of searching for a mixed NE is that their existence is more likely ensured by the linear dependence in $p(\boldsymbol{\epsilon})$ and hence a mixed NE is easier to find.

However, problem (6) is computationally much more expensive than problem (4) because the search space for the maximization part is much larger. In practice, this issue can be relaxed by approximating the optimal probability distribution in order to simplify the maximization process.

Here, we propose that the optimal distribution $p(\boldsymbol{\epsilon})$ can be approximated by exploiting adversarial samples found in the past rounds. In particular, we perform the maximization process in the same way as the above best-response approach and approximate the optimal probability distribution in the $(k+1)$-th round as

$$
p^{(k+1)}(\boldsymbol{\epsilon}) \approx \frac{1}{k} \sum_{j=1}^{k} \delta\left(\boldsymbol{\epsilon}-\boldsymbol{\epsilon}^{(j)}\right)
$$

using the historic adversarial samples $\boldsymbol{\epsilon}^{(1)}, \boldsymbol{\epsilon}^{(2)}, \cdots, \boldsymbol{\epsilon}^{(k)}$ in the past $k$ rounds. Consequently, in the following minimization process, 
the objective function based on the distribution (7) can be written as the average infidelity over the $k$ adversarial samples

$$
J\left[\boldsymbol{u}, B_{k}\right]=\frac{1}{k} \sum_{j=1}^{k} L\left[\boldsymbol{u}, \boldsymbol{\epsilon}^{(j)}\right],
$$

where $B_{k}=\left\{\boldsymbol{\epsilon}^{(1)}, \cdots, \boldsymbol{\epsilon}^{(k)}\right\}$.

In practice, the approximation (7) can be chosen more flexibly. For example, one does not have to use all historic adversarial samples in (8), because it will be too costly when $k$ is large and the early samples are likely less adversarial. Thus, we keep only the latest few samples, i.e., let the algorithm utilize only a finite number, say $s$, of adversarial samples (see Algorithm 1 for a summary). In this scenario, the originally discussed best-response approach can be taken as a special case with memory size being $s=1$.

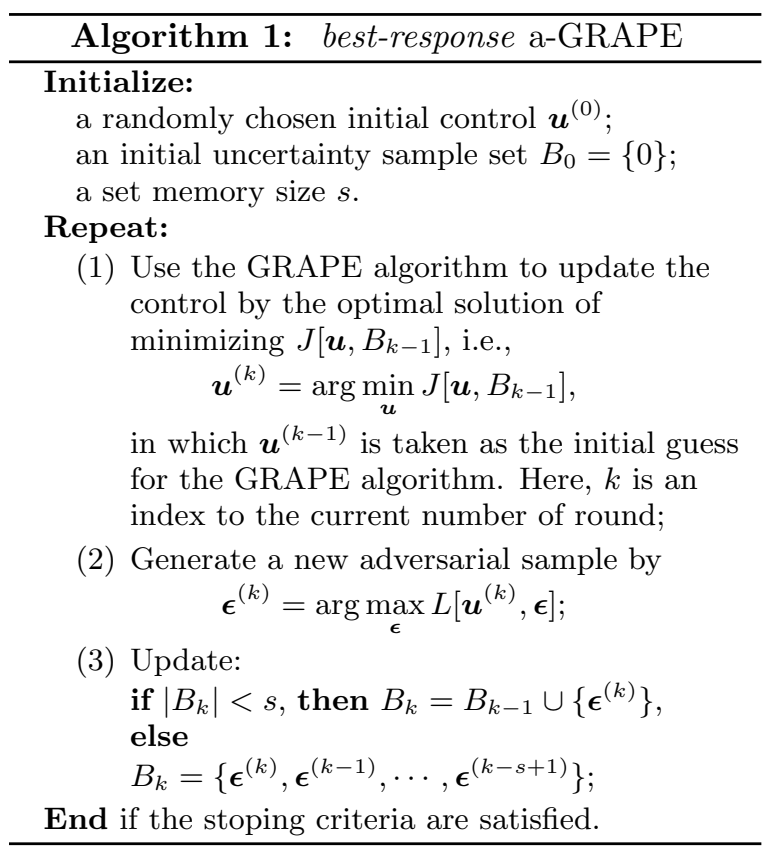

\section{B. Better-response approach}

In the above best-response approach, the minimization process is usually efficient as long as the control resources (e.g., bandwidth, pulse energy, etc.) are abundant, owing to the underlying nice control landscape topology over which almost all locally optimal controls are actually globally optimal [40, 41. However, the generation of adversarial samples is much harder because the maximization process is usually non-concave. Here, we relax this problem by choosing strong, but not necessarily the strongest adversarial samples for the training of robust controls. In this regard, we call this method a better-response approach.

The simplest way to search for better-response adversarial samples is to randomly choose a batch of uncertainty samples, calculate their corresponding cost, and keep the worst few members among them for the adversarial training in the next round (see Algorithm 2 for a description). The batch size of the samples should be sufficiently large so that the chosen adversarial samples have members close to the worst-case samples, but not too large to maintain computational efficiency. Naturally the better-response approach relying on random sampling usually takes more rounds of gaming, but each round can be much faster when the batch is not very large. Moreover, the randomness of sample batches in the better-response approach may bring additional benefits for the search to get away from unwanted false worst-case traps.

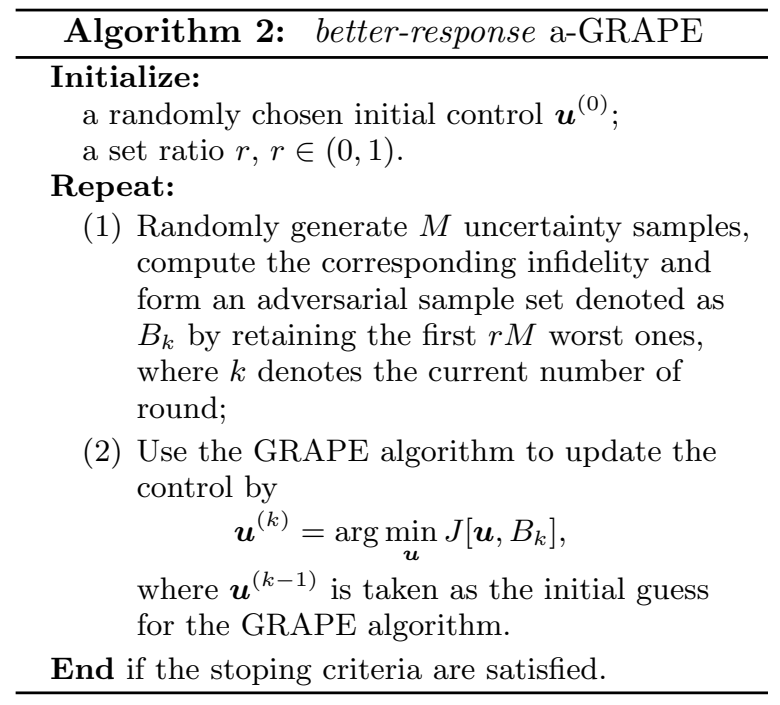

\section{SIMULATION RESULTS}

To illustrate the above game-based adversarial learning strategies for the robust control design, we simulate two quantum gate synthesis examples 
in this section. As will be seen in the simulations, the best-response approach can effectively suppress the worst-case performance, but does not always lead to good performance in high precision regimes, where the better-response approach is more effective. That is to say, the best-response and the better-response approaches do not mean the best performance and the better performance respectively, and which approach performs satisfactorily depends on case-specific requirements.

\section{A. Two-qubit system}

Consider a two-qubit quantum gate control problem with the following system Hamiltonian:

$$
\begin{aligned}
H(t)= & \left(1+\epsilon_{0}\right) g \sigma_{1 z} \otimes \sigma_{2 z}+\sum_{i=1}^{2}\left(1+\epsilon_{i}\right) \cdot \\
& {\left[u_{i x}(t) \sigma_{i x}+u_{i y}(t) \sigma_{i y}\right], }
\end{aligned}
$$

where $g=10 \mathrm{MHz}$ is the identified qubit-qubit coupling strength with $\epsilon_{0}$ being the identification error in the coupling constant; $\epsilon_{1}$ and $\epsilon_{2}$ represent the inhomogeneity of control fields. The dimensionless three uncertainty parameters are all assumed to be bounded by $\left|\epsilon_{i}\right| \leq 0.2$. In the simulation, the time duration of the control pulses is chosen as $T=300 \mathrm{~ns}$, which is evenly divided into 100 intervals over which the control fields are piecewise constant. The target $U_{f}$ is set as the controlled-NOT gate.

In the best-response approach, we apply a genetic algorithm to seek adversarial samples, and use $s=10$ historic adversarial samples to train the control function in each round. In the betterresponse approach, we uniformly generate $M=$ 100 random uncertainty samples in each round and keep the first $10 \%$ (i.e., $r=0.1$ ) worst ones as adversarial samples for training the control. Figure 1 shows the resulting learning curves, namely the achieved worst-case infidelity $L_{\max }$ versus the number of rounds, as well as the corresponding minimized average infidelity $J_{\min }$ over the selected adversarial samples versus the number of rounds. The robustness of the controls can be directly seen from the curves of worst-case infidelity, which are all enhanced during the optimization. The $L_{\max }{ }^{-}$ curve is initially far from the $J_{m i n}$-curve, but the gap is quickly reduced after several rounds of gaming. In the best-response approach, the gap is almost closed, showing that the optimized control (a) Best-response a-GRAPE

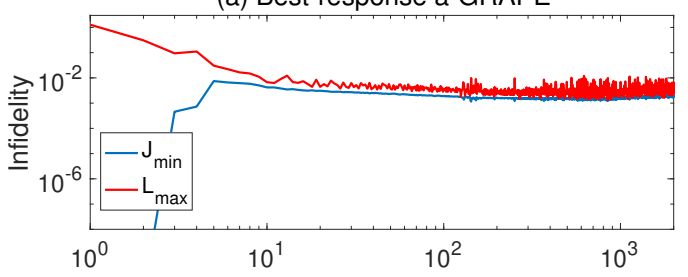

(b) Better-response a-GRAPE

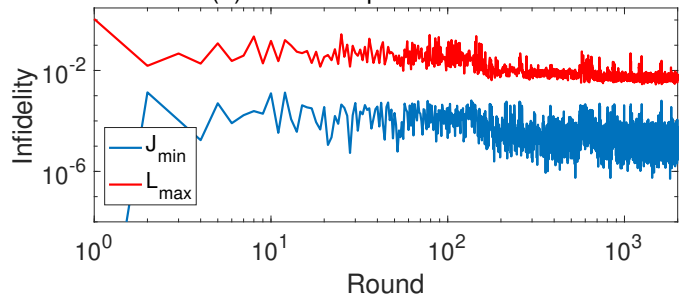

Fig 1: The learning curves of a-GRAPE for robust controls of the two-qubit system. The red curves correspond to the worst-case infidelity while the blue ones correspond to minimized average infidelity over selected adversarial samples in cases (a) the bestresponse approach with memory size $s=10$ and (b) the better-response approach with $M=100$ and $r=0.1$.

and uncertainty samples are likely close to a mixed NE. In the better-response approach, the gap still remains large after 2000 rounds. For both approaches, the control robustness is still enhanced by the game, with the (approximate) worst-case infidelity decreased to the level of $10^{-2}$.

As discussed above, neither the average performance nor the worst-case performance is the unique measure for quantifying the control robustness. To better evaluate the overall performance of an optimized control, we calculate and display the cumulative probability distribution function (cdf) $F(l)$ of the gate infidelity, i.e., the probability for the infidelity not being greater than $l$, in Fig. 2. Here, we also compare the a-GRAPE algorithms with the recently proposed b-GRAPE algorithm 21 (see Appendix for details) for robust control design subject to the average infidelity (i.e., Eq. (3p). In the simulations, the bGRAPE algorithm is run by 1 million iterations having the mini-batch size $n_{m b}=1$ and a learning rate $\alpha=0.002$, while the a-GRAPE algorithms are run by 844 rounds in the best-response approach and 1504 rounds in the better-response approach. 


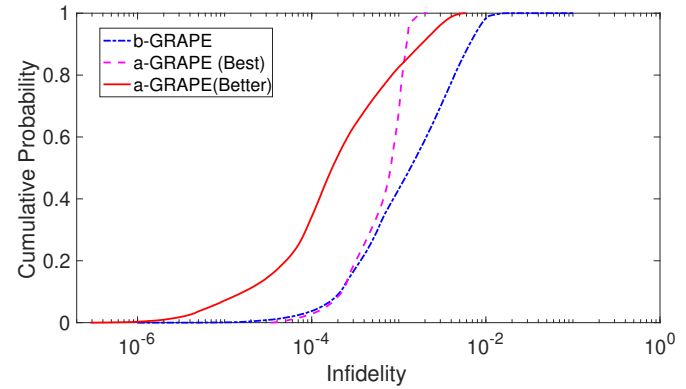

Fig 2: For the two-qubit system, the cumulative probability functions $F(l)$ of the gate infidelity $l$ under the controls optimized with b-GRAPE (batch size $n_{m b}=$ 1 ), best-response a-GRAPE (memory size $s=10$ ), and better-response a-GRAPE ( $M=100$ and $r=0.1$ ).

The cdf curve can be used to evaluate the control robustness from two perspectives. First, given a desired value of gate infidelity $l_{0}$ (e.g., the threshold error for quantum error correction), the cumulative probability $F\left(l_{0}\right)$ gives the confidence that the control can suppress the error below the value $l_{0}$. Second, given an expected confidence $F_{0}$ (say, $90 \%)$, the cdf can tell us at which threshold value (i.e., $l_{0}$ such that $F\left(l_{0}\right)=F_{0}$ ) the control can guarantee the confidence. As will be seen below, the robustness performance may vary at different levels of desired infidelity or expected confidence.

Figure 2 clearly shows that the controls optimized by the a-GRAPE algorithms, especially by the better-response approach, are much more robust as almost the entire cdf curves are higher than that generated with the b-GRAPE algorithm (i.e., with greater confidence at each value of infidelity). For example, as seen in Table I, the control optimized with the better-response a-GRAPE can suppress the gate error below $10^{-3}$ with a high confidence $82.5 \%$, while the control optimized with b-GRAPE has only $43.1 \%$ confidence. At the higher-precision level (i.e., infidelity lower than $\left.10^{-4}\right)$, the better-response approach still maintains $34.2 \%$ confidence, while the control optimized with b-GRAPE provides only $3.7 \%$ confidence. The performance of the best-response aGRAPE is only a little poorer than b-GRAPE in the high-precision regime, but much higher in the low-precision to medium-precision regime (i.e., infidelity in $10^{-3} \sim 10^{-2}$ ). Additionally, the bestresponse a-GRAPE achieves a lower worst-case in- fidelity than that achieved by the better-response a-GRAPE, which is consistent with the finding from the $L_{\max }$ curves in Fig. 1. However, the bestresponse a-GRAPE has poorer performance in the high-precision regime than the better-response a-GRAPE, which is also indicated by the $J_{\text {min }}$ curves displayed in Fig. 1. The comparison between different algorithms shows that there is no unique criterion for evaluating the control robustness. An optimized control may achieve satisfactory precision (e.g., infidelity in $10^{-3} \sim 10^{-2}$ ) over a large regime of uncertainties, but the highest precision it can achieve may be poor. In practice, one may need a balance between the high precision and the robust regions, especially using limited control resources.

\begin{tabular}{l|c|c|c}
\hline \hline & b-GRAPE & best-response & better-response \\
\hline $10^{-3}$ & $43.1 \%$ & $68.1 \%$ & $82.5 \%$ \\
$10^{-4}$ & $3.7 \%$ & $2.8 \%$ & $34.2 \%$ \\
\hline \hline
\end{tabular}

TABLE I: The two rows list the confidence for the gate infidelity to be below $10^{-3}$ and $10^{-4}$.

\section{B. Three-qubit system}

To see more clearly how the control performance relies on the uncertainties and algorithmic parameters, we simulate a three-qubit system with two uncertainty parameters, whose Hamiltonian is

$$
\begin{aligned}
H(t)= & J_{12}\left(1+\epsilon_{1}\right) \sigma_{1 z} \sigma_{2 z}+J_{23}\left(1+\epsilon_{2}\right) \sigma_{2 z} \sigma_{3 z} \\
& +\sum_{k=1}^{3}\left[u_{k x}(t) \sigma_{k x}+u_{k y}(t) \sigma_{k y}\right]
\end{aligned}
$$

where the nominal coupling constants are $J_{12}=$ $J_{23}=10 \mathrm{MHz}$. The uncertainty parameters $\epsilon_{1}$ and $\epsilon_{2}$ (i.e., identification errors of the coupling constants) are bounded by $\left|\epsilon_{i}\right| \leq 0.2$. In the simulation, the target unitary operation is selected as the Toffoli gate. The time period $[0, T]$, where $T=1 \mu \mathrm{s}$, is evenly divided into 100 intervals, over which the control fields are piecewise constant.

The previous illustration with the two-qubit system showed that the controls optimized by the aGRAPE algorithms could improve the worst-case performance, and here we want to see how the performance depends on the parameters, e.g., $s$ and $r$. We first compare the best-response a-GRAPE 


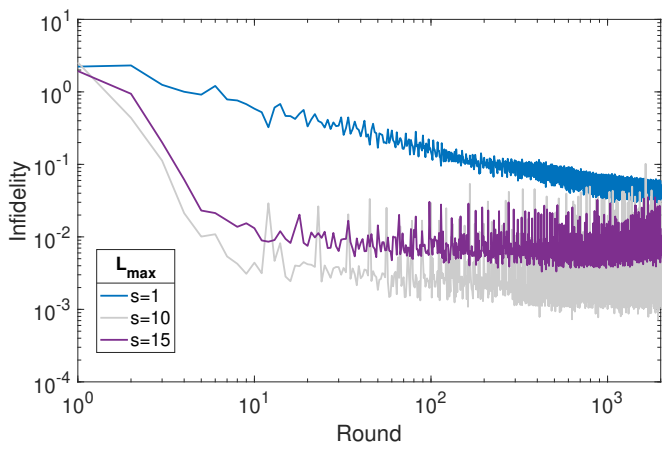

Fig 3: The worst-case infidelity versus the number of rounds in best-response a-GRAPE optimization of the three-qubit system with different memory sizes.

optimization processes with different memory sizes $s=1,10$, and 15 . The learning curves are shown in Fig. 3 in which the minimization curves are not displayed because the worst-case performance is only related with the maximization curve. It can be seen that the algorithm converges faster and finds more robust controls when using more, but not too many, historic adversarial samples. For example, the worst-case infidelity reaches $10^{-2}$ after only 7 rounds when $s=10$, which converges faster than the case $s=1$, and the worst infidelity is much lower. However, the case $s=15$ performs less satisfactorily than the case $s=10$. This is reasonable because elder historic samples tend to be less adversarial due to the fading memory effect.

For the better-response approach, we choose $M=100$ and compare the performance under $r=0.01,0.05$, and 0.1 , where the true worst-case infidelity in each round is estimated by 2000 independent random samples. Similar to the case of the best-response approach, the simulation results (see the learning curves in Fig. 4) show that the robustness of the optimized controls can be improved by using adequately many adversarial samples, but too many will not bring further improvement.

Since there are only two uncertainty parameters in this example, we can plot a 3D landscape to show how the infidelity varies with them, from which we can evaluate the overall robustness. In Fig. 5, we display 3D plots under controls optimized with the algorithms b-GRAPE (after 2 million iterations), best-response a-GRAPE (after 629 rounds) and better-response a-GRAPE (after 1986 rounds). The comparison shows that both
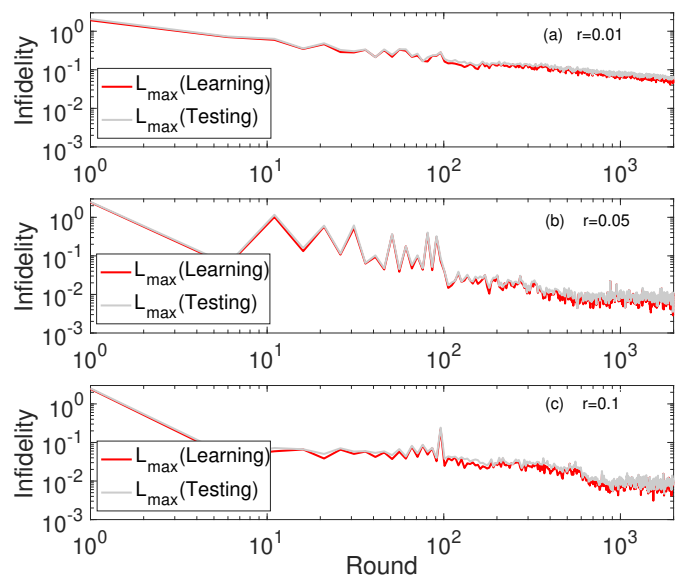

Fig 4: The worst-case infidelity versus the number of rounds in better-response a-GRAPE optimization of the three-qubit system with $r=0.01,0.05$ and 0.1 .

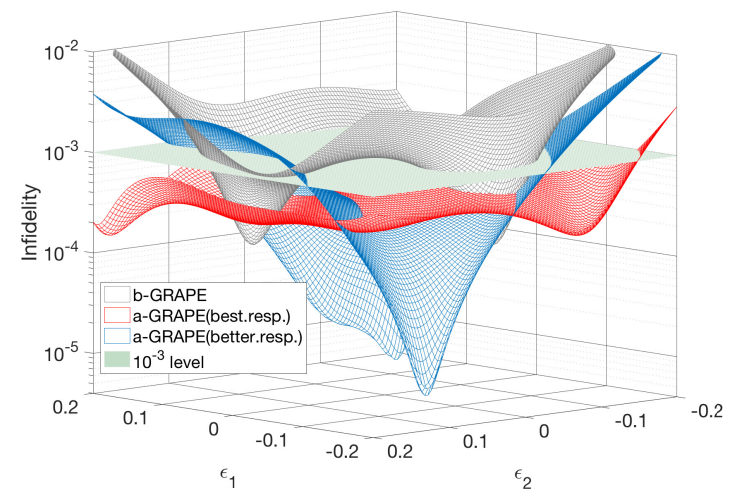

Fig 5: The infidelity versus uncertainty parameters under controls optimized with b-GRAPE with $n_{m b}=1$ and $\alpha=0.002$, best-response dynamic with memory size $s=10$, better-response approach with ratio $r=$ 0.05 respectively.

a-GRAPE algorithms outperform the b-GRAPE algorithm, as most of their landscape surfaces are below that of b-GRAPE. The best-response aGRAPE achieves the lowest worst-case infidelity and effectively suppresses almost the entire landscape down below the level of $L=10^{-3}$. However, its overall performance in the higher-precision regime (e.g., $L=10^{-4}$ ) is poorer than the betterresponse a-GRAPE. 
In addition to the best-response and betterresponse approaches, the a-GRAPE algorithms can be designed more flexibly such that it still works efficiently when the uncertainty vector $\epsilon$ is of large scale. For example, we may also perform the minimization process in a relaxed manner. As is described in Algorithm 3, we may update the control by only a few gradient-descent iterations (or stop at some prescribed error threshold) without having to reach the ultimate minimum, which responds better but not best to the adversarial samples. The maximization part can be done either with the best-response or better-response approaches. In this regard, the SCP algorithm 33 can be considered as a special case of the relaxed better-response approach with fixed sampled uncertainties and carefully selected learning rates. It is noteworthy that as the minimization and maximization are related to the fidelity and the robustness respectively, the balance between the two performance indexes can be adjusted flexibly via relaxing the two optimization processes.

To assess the feasibility of this idea, we apply the relaxed best-response (with $s=5, n=20$ and $m=20$ ) and relaxed better-response (with $r=0.25, n=30$ and $m=20$ ) a-GRAPE algorithms to the same three-qubit example. As is shown in Fig. 6, where the control robustness is evaluated by the cumulative probability functions, the relaxed a-GRAPE algorithms can also greatly outperform the b-GRAPE algorithm. Compared to their unrelaxed counterparts, the relaxed bestresponse and relaxed better-response a-GRAPE algorithms are not only faster, but also more robust in the high-precision regime (e.g., near the infidelity level $10^{-4}$ ).

\section{CONCLUSION}

We have proposed a family of adversarial learning algorithms, including the best-response and better-response approaches, for the robust control design of quantum systems. The algorithms are subject to the optimization of worst-case gate infidelity, which can be treated and resolved from a game-theoretic perspective. Numerical simulations show that these a-GRAPE algorithms can achieve high control robustness. In particular, the best-response approach can effectively suppress the error over a larger domain at a satisfactory level of precision, but in the extremely high-
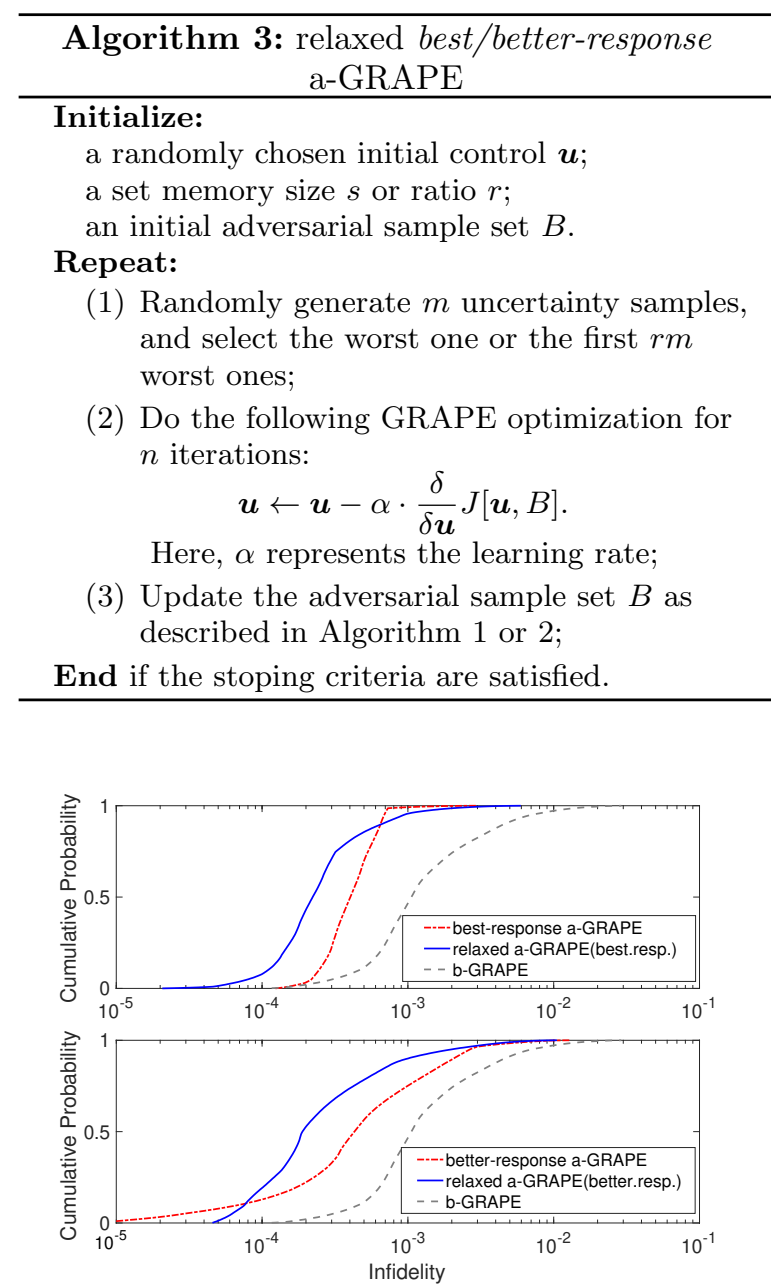

Fig 6: The cumulative probability versus infidelity under the corresponding controls used in Fig. 5 and the ones obtained from the relaxed best-response approach run by 10995 rounds and the relaxed better-response algorithm run by 3850 rounds.

precision regime, the better-response approach is superior. Both approaches have their regimes of practical utility depending on the applicationspecific requirement of the control. We also demonstrate that a family of a-GRAPE algorithms can be expanded by relaxing the maximization and minimization processes.

It should be noted that, although a-GRAPE usually outperforms b-GRAPE, the computational burden is also heavier, and the tuning of algorithm parameters (e.g., memory sizes, batch sizes, ratios 
or learning rates) is application-specific. Further studies are needed to deduce how to optimize the choices of these parameters, or even in an adaptive fashion.

As we remarked, we did not require on the existence of Nash equilibria (NE) as an appropriate adversarial algorithm can enhance the robustness no matter whether the NE exists or not. From our simulations, it appears that a mixed NE is more likely approached in the best-response a-GRAPE with a larger memory size. From a theoretical perspective, a better understanding of the existence of a NE will be useful to attain. This topic will be explored in future studies.

\section{Acknowledgments}

The author Rebing $\mathrm{Wu}$ acknowledges the support of the National Key R\&D Program of China (Grants No. 2018YFA0306703 and No. 2017YFA0304304) and NSFC (Grants No. 61833010 and No. 61773232). The author Herschel Rabitz acknowleges the support of the National Science Foundation (CHE-1763198).

\section{Appendix: b-GRAPE algorithm}

The b-GRAPE algorithm presented in [21] is a stochastic gradient algorithm. The optimization process follows the gradient evaluated with randomly chosen batches of samples, so that the uncertainties can be effectively used to improve the robustness. Here, "b" stands the "batch". The b-GRAPE algorithm is described in Algorithm 4 .
[1] Davide Castelvecchi. Quantum computers ready to leap out of the lab in 2017. Nature, 541(7635):9-10, 2017.

[2] X. Dai, Y. Deng, X. Peng, and Y. Jin. Quantumdot light-emitting diodes for large-area displays: Towards the dawn of commercialization. Advanced Materials, 29(14):1607022, 2017.

[3] Masoud Mohseni, Peter Read, Hartmut Neven, Sergio Boixo, and Vasil Denchev. Commercialize early quantum technologies. Nature, 543(7644):171-174, 2017.

[4] Michael A Nielsen and Isaac Chuang. Quantum computation and quantum information. AAPT, 2002.

[5] Navin Khaneja, Timo Reiss, Cindie Kehlet, Thomas Schulte-Herbrüggen, and Steffen J. Glaser. Optimal control of coupled spin dynamics: design of nmr pulse sequences by gradient ascent algorithms. Journal of Magnetic Resonance, 172(2):296-305, 2005.

[6] Mogens Dalgaard, Felix Motzoi, Jens Jakob Sorensen, and Jacob Sherson. Global optimization of quantum dynamics with alphazero deep exploration. arXiv preprint arXiv:1907.05672, 2019.

[7] K. Bergmann, H. Theuer, and B. W. Shore. Coherent population transfer among quantum states of atoms and molecules. Reviews of Modern Physics, 70(3):1003, 1998.

[8] Klaas Bergmann, Nikolay V. Vitanov, and Bruce W. Shore. Perspective: Stimulated raman adiabatic passage: The status after 25 years.

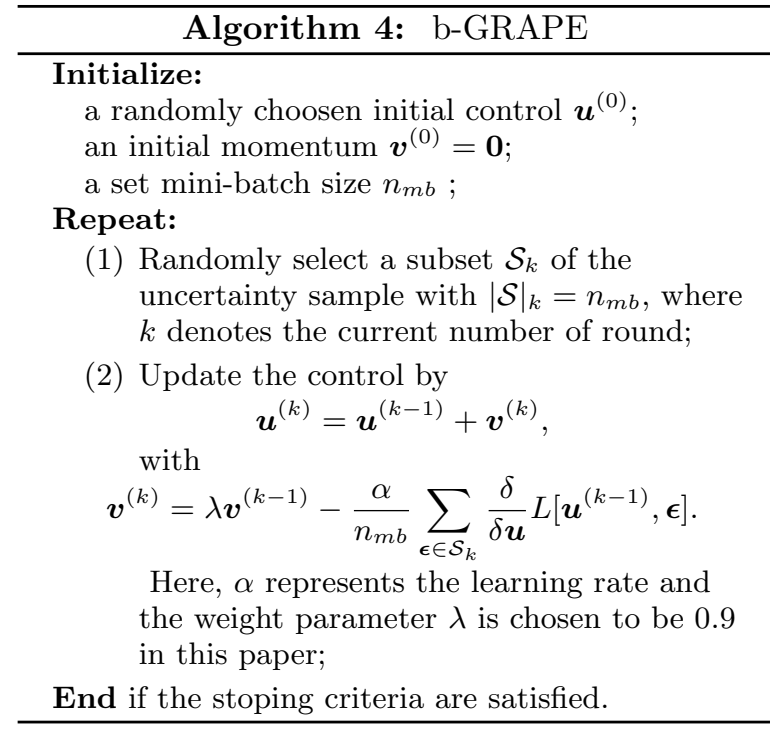

The Journal of chemical physics, 142(17):170901, 2015.

[9] Nikolay V. Vitanov, Andon A. Rangelov, Bruce W. Shore, and Klaas Bergmann. Stimulated raman adiabatic passage in physics, chemistry, and beyond. Reviews of Modern Physics, 89(1):015006, 2017.

[10] Lorenza Viola, Emanuel Knill, and Seth Lloyd. 
Dynamical decoupling of open quantum systems. Physical Review Letters, 82(12):2417-2421, 1999.

[11] Kaveh Khodjasteh and Lorenza Viola. Dynamically error-corrected gates for universal quantum computation. Physical review letters, 102(8):080501, 2009.

[12] Kaveh Khodjasteh and Daniel A Lidar. Performance of deterministic dynamical decoupling schemes: Concatenated and periodic pulse sequences. Physical Review A, 75(6):062310, 2007.

[13] K. Khodjasteh and D. A. Lidar. Fault-tolerant quantum dynamical decoupling. Physical Review Letters, 95(18):180501, 2005.

[14] Ehsan Zahedinejad, Joydip Ghosh, and Barry C. Sanders. Designing high-fidelity single-shot threequbit gates: A machine-learning approach. Physical Review Applied, 6(5):054005, 2016.

[15] Zahedinejad Ehsan, Ghosh Joydip, and Barry C. Sanders. High-fidelity single-shot toffoli gate via quantum control. Physical Review Letters, 114(20):200502, 2015.

[16] Jr-Shin Li and Navin Khaneja. Control of inhomogeneous quantum ensembles. Physical review A, 73(3):030302, 2006.

[17] Jun Zhang, Loren Greenman, Xiaotian Deng, and K. Birgitta Whaley. Robust control pulses design for electron shuttling in solid-state devices. IEEE Transactions on Control Systems Technology, 22(6):2354-2359, 2014.

[18] Chunlin Chen, Daoyi Dong, Ruixing Long, Ian R Petersen, and Herschel A Rabitz. Sampling-based learning control of inhomogeneous quantum ensembles. Physical Review A, 89(2):023402, 2014.

[19] Daoyi Dong, Mohamed A. Mabrok, Ian R. Petersen, Qi Bo, Chunlin Chen, and Herschel Rabitz. Sampling-based learning control for quantum systems with uncertainties. IEEE Transactions on Control Systems Technology, 23(6):2155-2166, 2013.

[20] Chengzhi Wu, Bo Qi, Chunlin Chen, and Daoyi Dong. Robust learning control design for quantum unitary transformations. IEEE transactions on cybernetics, 47(12):4405-4417, 2017.

[21] Rebing Wu, Haijin Ding, Daoyi Dong, and Xiaoting Wang. Learning robust and highprecision quantum controls. Physical Review A, 99(4):042327, 2019.

[22] Hai-Jin Ding and Re-Bing Wu. Robust quantum control against clock noises in multiqubit systems. Physical Review A, 100(2):022302, 2019.

[23] A. A Stoorvogel. The robust h/sub 2/control problem: a worst-case design. IEEE Transactions on Automatic Control, 38(9):1358-1371, 1993.

[24] Myung Gon Yoon, Valery A. Ugrinovskii, and Ian R. Petersen. On the worst-case disturbance of minimax optimal control. Automatica, 41(5):847$855,2005$.
[25] Peng Shi. Gain-scheduled worst-case control on nonlinear stochastic systems subject to actuator saturation and unknown information. Journal of Optimization Theory \& Applications, 156(3):844858, 2013.

[26] A. S. Poznyak, T. E. Duncan, B. Pasik-Duncan, and V. G. Boltyansky. Robust stochastic maximum principle for multi-model worst case optimization. International Journal of Control, 75(13):1032-1048, 2002.

[27] Johan G. B. Beumee and Herschel Rabitz. Robust optimal control theory for selective vibrational excitation in molecules: A worst case analysis. Journal of Chemical Physics, 97(2):1353-1364, 1992.

[28] Hong Zhang and Herschel Rabitz. Robust optimal control of quantum molecular systems in the presence of disturbances and uncertainties. Physical Review A, 49(4):2241, 1994.

[29] Andy Koswara and Raj Chakrabarti. Robustness of controlled quantum dynamics. Phys.rev.a, 90(4), 2014.

[30] M. R. James, H. I. Nurdin, and I. R. Petersen. $h_{\infty}$ control of linear quantum stochastic systems. IEEE Transactions on Automatic Control, 53(8):1787-1803, 2007.

[31] A. I. Maalouf and I. R. Petersen. Time-varying $h_{\infty}$ control for a class of linear quantum systems: A dynamic game approach. Automatic, 48(11):2908-2916, 2012.

[32] Aline I. Maalouf and Ian R. Petersen. Finite horizon $h_{\infty}$ control for a class of sampled-data linear quantum systems. International Journal of Robust \& Nonlinear Control, 27(13), 2017.

[33] Robert L. Kosut, Matthew D. Grace, and Constantin Brif. Robust control of quantum gates via sequential convex programming. Physical Review A, 88(5):052326, 2013.

[34] John Von Neumann, Oskar Morgenstern, and Harold William Kuhn. Theory of games and economic behavior. Princeton university press, 2007.

[35] Christian Szegedy, Wojciech Zaremba, Ilya Sutskever, Joan Bruna, Dumitru Erhan, Ian Goodfellow, and Rob Fergus. Intriguing properties of neural networks. arXiv preprint arXiv:1312.6199, 2013.

[36] Uri Shaham, Yutaro Yamada, and Sahand Negahban. Understanding adversarial training: Increasing local stability of supervised models through robust optimization. Neurocomputing, 307:195$204,2018$.

[37] Ian J Goodfellow, Jonathon Shlens, and Christian Szegedy. Explaining and harnessing adversarial examples. arXiv preprint arXiv:1412.6572, 2014.

[38] Ian Goodfellow, Jean Pouget-Abadie, Mehdi Mirza, Bing Xu, David Warde-Farley, Sherjil Ozair, Aaron Courville, and Yoshua Bengio. Generative adversarial nets. In Advances in neural 
information processing systems, pages 2672-2680, 2014.

[39] Drew Fudenberg and David K. Levine. The theory of learning in games, volume 2. MIT press, 1998.

[40] Herschel Rabitz, Michael Hsieh, and Carey Rosenthal. Landscape for optimal control of quantum- mechanical unitary transformations. Physical Review A, 72(5):052337, 2005.

[41] Benjamin Russell, Herschel Rabitz, and Rebing Wu. Quantum control landscapes are almost always trap free. arXiv preprint arXiv:1608.06198, 2016. 